\title{
On the non-thermal effects of electromagnetic fields on charge transport processes in ecosystems
}

\author{
L. Brizhik \\ Bogolyubov Institute for Theoretical Physics, Kiev, Ukraine
}

\begin{abstract}
It is shown that periodic electromagnetic (EM) fields cause non-thermal effects on nonlinear charge transport processes in living organisms and ecosystems. In particular, such processes take place during respiration or photosynthesis and in hydrogen-bonded water chains present in interfacial water in cells, in coherence domains of water in the atmosphere and oceans. The nonlinear charge transport is mediated by solitons and bisolitons which are formed by single charge (electron, proton) or, respectively, two electrons in a singlet state, that are selftrapped in the self-induced local distortion of macromolecules or water chains. Due to oscillations of their velocity caused by discreteness of chains, (bi)solitons emit EM radiation of characteristic frequencies, determined by the intensity of the redox processes. In the presence of the periodic field (bi)solitons attain additional oscillations, and, therefore, the frequency of their EM radiation becomes modified, which affects redox processes in particular and the whole metabolism in general. It is shown that (bi)solitons have some characteristic frequencies, with respect to which periodic EM fields have non-thermal resonant effect on their dynamics and stability. Relatively strong enough unbiased periodic EM radiation can cause drift of (bi)solitons in molecular chains, and, therefore, it affects charge transport processes. These results show that changes in the EM solar activity can have effects on metabolism of living organisms and functioning of ecosystems and indicate a possible danger of the EM smog in ecosystems.
\end{abstract}

Keywords: ecosystems, soliton, bisoliton, charge transport, electromagnetic field, ratchet dynamics, water, electromagnetic smog. 


\section{Introduction}

The existence of complex dynamical systems, such as ecosystems, implies exchange between the sub-units of the system not only with matter and energy, but what is even more important, also with information. While the first two exchange processes are intact, short-range, the exchange with information is a long-range process, which should occur very fast to provide the effective stability in the dynamical change of the conditions that are considered as an environment for sub-units. Here the question arises: what is the messenger for such fast long-range exchange with information? Our hypothesis (see Brizhik et al. $[1,2])$ is that it is the electromagnetic field (EM), and here we develop further this theory. We show that such EM field not only is produced in the processes that occur in the system, but it also regulates these processes in the self-consistent way, providing thus the dynamical stability of the system and the emergence of the hierarchy of mutually interacting ecosystems. These interactions in and between sub-units are mediated by water which plays an essential role in all these processes due to its very peculiar and unique properties (see, e.g., Del Giudice and Tedeschi [3], Pollack [4], Voeikov [5]).

Electromagnetic field (EMF) is responsible for the matter formation and its properties, both in non-animated and alive, as well as in complex systems comprising them both, such as ecosystems. It is also the main tool of information storage and transfer in modern technologies. Till late 80 s of the last century EMF was studied and technologically applied in linear systems whose optical properties almost do not depend on the field intensity. Moreover, in most cases EMF was assumed to be an external field for the problems studied. This is often the case, indeed. Nevertheless, there is a large class of systems which change the properties of the field itself, and systems with endogenous EMF, when the field itself is an intrinsic property of the system and is dynamically self-consistent with it. An important example of such systems is living organisms whose endogenous EMF is essential not only for their functioning, but provides their whole metabolism. No surprise that external EMF affects such systems, and that the caused bioeffects are very complicated and intensity and frequency dependent. In this paper we study the effect of EMF on soliton mediated charge transport in nonlinear systems, such as living organisms and water chains (Davydov [6], Antonchenko et al. [7]) or coherence domains of water (Del Giudice [8] and references therein). It is worth to recall here that term 'soliton' means a localized solitary wave solution of nonlinear equation(s), which is stable due to mutual compensation of nonlinearity and dispersion, and which is stable with respect to external perturbations and collisions with similar solitons. In the context of present paper solitons are charge carriers in macromolecules or hydrogen-bonded chains of water molecules, bound with the self-induced chain deformation (see Davydov [6]). In Section 2 we describe briefly main properties of solitons and bisolitons, and in Section 3 we study dynamics of solitons and bisolitons in periodic EMF. 


\section{Main characteristics of solitons and bisolitons}

It is known that charge transport accompanies redox process in organisms with photosynthetic or respiratory system. In such organisms certain parts of the charge transport chain (so called Krebs cycle) in redox processes involve macromolecular enzymes which form alpha-helical conformation consisting of three almost parallel polypeptide chains, stabilized by hydrogen bonds along them. According to Davydov [6], charge transport along these enzymes is provided by soliton mechanism, when electrons released at the initial stage of the photosynthesis or respiration, cause deformation of the macromolecule and get self-trapped in soliton states. Similarly, solitons are formed in hydrogen bonded water molecules (see Davydov [6], Antonchenko et al. [7]). The Hamiltonian of a molecular chain with extra charge carriers consists of three terms, which are the Hamiltonians of free charge carriers, of the lattice and their interaction. In the site representation such Hamiltonian leads to the system of nonlinear coupled equations, which can be solved numerically or studied analytically in the continuum approximation, when discrete index $n$ which numerates unit cells in a molecular chain is substituted by a continuous variable $x=n a$. Here $a$ is a lattice constant (distance between the nearest molecules). In a particular case of hydrogen bounded polypeptide chain in macromolecules with one or two extra charge carriers with opposite spins the resulting system of equations can be reduced to the nonlinear Schroedinger equation (see Davydov [6], Brizhik and Davydov [9]):

$$
i \hbar \frac{\partial \Psi(x, t)}{\partial t}=-J \frac{\partial^{2} \Psi(x, t)}{\partial x^{2}}-2 J G|\Psi(x, t)|^{2} \Psi(x, t) .
$$

Here $\Psi(x, t)$ is the wave function of a charge carrier which determines the amplitude of the probability of the charge presence at position $x$ at time moment $t, \hbar$ is the Planck constant, $J$ is the electron resonant interaction energy between the nearest sites, and $\mathrm{G}$ is the nonlinearity constant. The nonlinear term in eqn. (1) is the deformational potential which accounts the deformation of the chain induced by charges due to the electron-lattice interaction. The nonlinearity constant $G$ in eqn. (1) for one $\left(G=G_{s}\right)$ or two charge carriers in a singlet state $\left(G=G_{b s}\right)$ takes the following values

$$
G_{s}=g, G_{b s}=2 g,
$$

respectively, where $g$ is the dimensionless electron-lattice coupling constant:

$$
g=\frac{\chi^{2}}{2 J w\left(1-s^{2}\right)}, s^{2}=\frac{V^{2}}{V_{s}^{2}} .
$$

In eqn. (3) $\chi$ is the strength of electron coupling with the lattice, $w$ is the elasticity of the chain, $V$ is the velocity of the charge carrier, and $\mathrm{V}_{\mathrm{s}}$ is sound velocity in the chain.

Eqn. (1) admits normalized to unity soliton-type solution 


$$
\Psi(x, t)=\frac{1}{2} \sqrt{G} \frac{\exp (i k x-i E t / \hbar)}{\cosh \{G[x-R(t)] / 2 a\}},
$$

where $E$ is the energy, $R(t)=V t$ is the c.m. coordinate of the charge carrier, $k$ is its wave-vector. In the case of one extra charge carrier it is called soliton, and in the case of two charge carriers with opposite spins solution (4) describes bisoliton.

Thus, soliton is a bound state of a charge carrier (electron or proton) with self-induced local deformation of the chain. As any solitons in other nonlinear systems, solitons in molecular chains are exceptionally stable due to the compensation of wave dispersion and nonlinearity, their energy is lower than the energy of free charge carriers, they propagate along the molecular chain with the velocity, lower than the sound velocity in the system, and as a result, they can propagate on macroscopically large distances with very low energy dissipation. From eqns. (2)-(4) we conclude, that the width of the bisoliton is twice smaller than the width of a soliton:

$$
l_{s}=\frac{2 \pi}{g}, l_{b s}=\frac{\pi}{g} .
$$

The energy of the system, including the energy of the chain deformation, is lower than the energy of free charge carriers, $E_{0(s, b s)}$, and is given below:

$$
E_{s}(V=0)=E_{0(s)}-J \frac{g^{2}}{12}, E_{b s}(V=0)=E_{0(b s)}-\frac{2}{3} J g^{2} .
$$

Therefore, the binding energy of two charge carriers in the bisoliton state is

$$
E_{\text {bind }}(V=0)=\frac{1}{2} J^{2} \text {. }
$$

The effective mass of a (bi)soliton is bigger than the mass of free charge carrier(s), since it includes also the effective mass of the chain deformation, and is determined below for a soliton and bisolton states

$$
\begin{gathered}
M_{s}=m+M_{d e f(s)}, M_{d e f(s)}=\frac{m M \chi^{4}}{6 \hbar^{2} w^{3}}, \\
M_{b s}=m+M_{\text {def }(b s)}, M_{d e f(b s)}=\frac{4 m M \chi^{4}}{3 \hbar^{2} w^{3}},
\end{gathered}
$$

respectively. Here $m$ is the mass of a free charge carrier, and $M$ is the mass of a molecule in a chain.

\section{Effect of EMF on (bi)solitons}

It has been shown by Brizhik et al. [10] that solitons have some characteristic frequency

$$
\omega_{s}=g \frac{V_{S}}{\pi},
$$


which is determined as inverse time, necessary for the sound to pass the width of the soliton localization. Taking into account eqn. (5), we see that the characteristic frequency of a bisoliton is twice bigger than that of a soliton:

$$
\omega_{b s}=2 g \frac{V_{s}}{\pi} .
$$

According to Brizhik et al. [10], the external periodic EMF has a resonant effect on the dynamics of a soliton with respect to its characteristic frequency (10). Similar situation takes place for bisolitons. Namely, in the oscillating fields (bi)solitons start to oscillate with the frequency of the field $\omega$ as quasi-particles in the field of external periodic force. In low-frequency (at $\omega<\omega_{(s, b s)}$ ) and high frequency fields (at $\omega>\omega_{(s, b s)}$ ) (bi)olitons are stable, while at the resonant frequency $\omega=\omega_{(s, b s)}$ they intensively absorb energy from the field, and radiate it in the form of sound waves and EM radiation, rapidly decaying from a (bi)soliton state into the state of delocalized free charge carriers. Of course, such changes affect the efficiency of the redox processes and, therefore, the whole metabolism of the living organism or functioning of the corresponding ecosystem.

Another aspect of the effects of EMF on (bi)solitons is related to the so-called ratchet phenomenon, which consists in the ability of an excitation to attain a nonzero velocity (start to drift in the field of deterministic or stochastic unbiased (zero mean-value) periodic in time forces (see review by Reinmann [11]). Ratchet dynamics of solitons in nonlinear systems, described by nonlinear equations, such as sine-Gordon, was proven numerically (see, e.g., Zolotaryuk and Salerno [12], Gorbach et al. [13]). Also it has been shown by Brizhik et al. [14] that charged solitons can drift in molecular chains in the non-biased a.c. EMF. Appearance of such uni-directed motion is a critical phenomenon with respect to the intensity of the field and its frequency. This phenomenon can be studied by computer simulations of the coupled system of nonlinear discrete equations describing charge carriers in a deformable molecular chain. Some aspects of the ratchet dynamics can be described also within the approach of collective coordinates, taking into account the presence of the periodical in space lattice potential $U(R)$ (so-called Peierls-Nabarro potential). In this latter case (bi)soliton dynamics in the external field $E(t)$ with account of the energy dissipation has the form

$$
M_{s(b s)} \frac{d^{2} R}{d t^{2}}=\gamma \frac{d R}{d t}-\frac{d U(R)}{d R}+q E(t),
$$

where effective mass of a (bi)soliton is determined in eqns. (8)-(9), $\gamma$ is the friction coefficient, and $q$ is the charge of a particle ( $q=e$ for a soliton, $q=2 e$ for a bisoliton, $e$ is the elementary charge). In the simplest case the lattice potential is represented as a double-well periodic potential

$$
U(R)=U_{1} \cos \left(2 \pi \frac{R}{a}\right)+U_{2} \cos \left(4 \pi \frac{R}{a}+\varphi\right)
$$


where $U_{1}$ and $U_{2}$ are the heights of the harmonics in a double-well potential, and $\varphi$ accounts their asymmetry. An example of such soliton drift in a harmonic unbiased field $E(t)=E_{0} \sin (2 \pi t / T)$ for the intensity of the field $E_{0}=8.7$ and period $T=2000$ is shown in fig 1 (the intensity of the field is measured in units $e a / J$ and time in units of $\hbar / J$ ).

As it has been shown in Brizhik et al. [14], such drift takes place only when the intensity of the field exceeds some critical value, which depends on the parameter values of the system, and in the fields whose frequency is not too high, namely, if it is less than the characteristic frequency of (bi)soliton (10) or (11), respectively. These conditions are necessary to allow (bi)soliton to overcome the potential barrier and the velocity (and frequency of oscillations) should be low enough so that the chain deformation could follow fast dynamics of electrons.

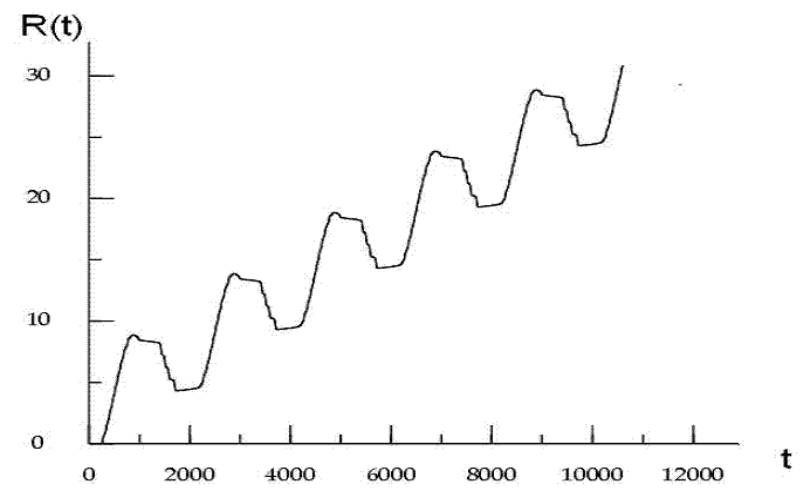

Figure 1: Position of the c.m.c. of a soliton, $R(t)$, as function of time, $t$, in the harmonic field with the parameters $\mathrm{E}_{0}=8.7, \mathrm{~T}=2000$ at $\gamma=400$. Parameters of the double-well potential in eqn. (13) are $\mathrm{U}_{1}=1$, $\mathrm{U}_{2}=0.25, \varphi=-1.25$.

\section{Conclusions}

Present study shows that external EMFs even of small intensities modify dynamics of charge carriers in living organisms and ecosystems, and that these effects depend on the frequency of the field. According to Davydov [6], charge transport in redox processes in living organisms and water systems is mediated by solitons and their bound states (bisolitons). In the presence of an oscillating EMF (bi)solitons oscillate with the frequency of the field, and the effective mass of (bi)soliton is a non-monotonous function of the frequency. At the resonant frequency (bi)solitons are unstable, they decay into unbound states of free electrons and lattice deformation.

The very fact, that (bi)solitons start to oscillate in the external periodic field, is very important, since during such oscillations (bi)solitons excite vibrational 
modes in a corresponding chain. Moreover, these oscillations, according to the Maxwell equations, will result in the emission by (bi)solitons of their own EMF. According to Brizhik et al. [15], frequency of the main harmonic of this radiation is determined by the average velocity of the soliton. In the absence of the field such radiation is caused by the discreteness of molecular chains and adds up to the endogenous EMF of the system.

Via exchange with the electromagnetic radiation, (bi)solitons get synchronized, and as a result, the system can attain coherence. This electromagnetic radiation is the fast messenger in the information exchange between the subunits of the complex system (cells, organs, individuals, ecosystems) which leads to the appearance of the long-range order in the system. The non-local self-consistent dynamics of matter and EMF is able to provide the development of dissipative structures, increasing the network of individuals, processes, and information and resulting in the appearance of the hierarchy of dissipative systems able to support fast and long-range exchange of the information and interconnectedness of the system (see Prigogine and Nicolis [16]).

In the non-biased EMF (bi)solitons can attain additional drift due to the ratchet effect. In this case their velocity is not just the velocity, determined by the rate of redox processes, but includes also the drift velocity. Therefore, the appearance of the drift modifies frequency of the (bi)soliton radiation, i.e., modifies intrinsic EMF of the system. This, of course, affects redox processes, and in such a way, metabolism of the living organism or functioning of an ecosystem. White noise can also result in the ratchet effect, as it has been shown by Luczka et al. [17], Bartussek et al. [18], Gomez-Marin and Sancho [19]. Therefore, we can expect that noise can also cause uni-directed current of (bi)solitons in molecular systems. This suggests a possible mechanism of the effects of the solar activity on biosystems via its influence on the nonlinear charge transport processes. It follows from these results technologically produced electromagnetic smog even of low intensities can be potentially dangerous, since, as we have shown above, the bioeffects of oscillating fields have resonant nature, and, therefore, are non-thermal. Finally, we remark that living systems and ecosystems, are not only complex open systems, they are also ontic, (see Tiezzi et al. [20]) so that although each sub-system is protected against excessive external influences, it remains open, which allows functioning of its complex internal organization in fast varying conditions. Such openness is not only because of the exchange with matter and energy, but also with the information, which occurs via self-consistent (endogenous) electromagnetic field.

\section{Acknowledgements}

I express my sincere gratitude to Prof. E. Del Giudice for stimulating discussions and co-operation. I acknowledge Prof. W. J. Zakrzewski and Drs. A. A. Eremko and B. M. A. G. Piette for our joint research, the results of which made this paper possible. I also acknowledge the Organizers of the ECOSUD Conference for the 
financial support, thanks to which I got the possibility to attend the event, which was so important to my friend and co-author Enzo Tiezzi, to whose memory I dedicate this contribution.

\section{References}

[1] Brizhik L., Del Giudice E., Jorgensen S.E., Marchettini N., and Tiezzi E. The role of electromagnetic potentials in the evolutionary dynamics of ecosystems. Ecological Modelling, 220, pp. 1865-1869, 2009.

[2] Brizhik, L., Del Giudice, E., Tedeschi, A., Voeikov, V., The role of water in the information exchange between the components of an ecosystem, submitted to Ecological Modelling, 2011.

[3] Del Giudice, E.; Tedeschi, A., Water and the autocatalysis in living matter. Electromagn. Biol. Med., 28, pp 46-54, 2009.

[4] Pollack, G.H., Water, energy and life: Fresh views from the water's edge. Int. J. Des. Nat. Ecodyn. 5, pp 27-29, 2010.

[5] Voeikov, VL. Fundamental Role of Water in Bioenergetics. In: Biophotonics and Coherent Systems in Biology. Beloussov, L.V., Voeikov, V.L., Martynyuk, V.S., editors. Springer, N.-Y., 2007, pp 89-104.

[6] Davydov, A.S., Solitons in Molecular Systems (2nd ed.), Reidel, Dordrecht, 1985.

[7] Antonchenko, V.Ya., Davydov, A.S., Zolotaryuk, A.V., Solitons and proton motion in ice-like structure, Phys. Stat. Sol. (b) 115, pp 945-952, 1983.

[8] Del Giudice, E. Old and new views on the structure of matter and the special case of living matter, J. Phys.: Conf. Series; 67, 012006, 2006.

[9] Brizhik, L.S., Davydov, A.S., The electrosoliton pairing in soft molecular chains, Low Temp. Phys., 10, pp 748-753, 1984.

[10] Brizhik, L., Cruzeiro-Hansson, L., Eremko A., Influence of electromagnetic radiation on molecular solitons, J. Biol. Phys., 24, pp 19-39, 1998.

[11] Reimann, P., Brownian motors: noisy transport far from equilibrium, Phys. Rep., 361, pp 57-265, 2002.

[12] Zolotaryuk, Y., Salerno, M., Discrete soliton ratchets driven by biharmonic fields, Phys. Rev. E 73, 066621 (12 pages), 2006.

[13] Gorbach, A.V., Denisov, S., Flach, S., Optical ratchets with discrete cavity solitons, Opt. Lett., 31, pp 1702-1704, 2006.

[14] Brizhik, L., Eremko, A., Piette, B., Zakrzewski, W., Ratchet dynamics of large polarons in asymmetric diatomic molecular chains. J. Phys.:Cond. Matt., 22, 155105, 9 pp., 2010.

[15] Brizhik, L., Eremko, A., Nonlinear model of the origin of endogenous alternating electromagnetic fields and self regulation of metabolic processes in biosystems, Electromagnetic Biol. and Med., 22, pp 31-39, 2003.

[16] Prigogine, I., Nicolis, G., Self-Organization in Non-Equilibrium Systems. Wiley, New York, 1997.

[17] Luczka, J., Bartussek, R., Hänggi, P., White-noise-induced transport in periodic structures, Europhys. Lett., 31(8), pp 431-436, 1995. 
[18] Bartussek R., Hänggi P., Lindner, B., Schimansky-Geier, L., Ratchets driven by harmonic and white noise, Physica D, 109, pp 17-23, 1997.

[19] Gomez-Marin, A., and Sancho, J.M., Brownian pump powered by a whitenoise flashing ratchet. Phys. Rev. E, 77, 031108, 2008.

[20] Tiezzi, E., Cecconi, G., Marchettini, N., Confined ontic open systems. Int. J. of Design and Nature and Ecodynamics, 5, 3-9, 2010. 